Polymer Journal, Vol. 23, No. 4, pp 311-320 (1991)

\title{
Structure and Properties of Fatigued Segmented Poly(urethaneurea)s IV. Dynamic Infrared Dichroism
}

\author{
Tomoyuki Yamamoto, Mitsuhiro Shibayama, and Shunji Nomura* \\ Department of Polymer Science and Engineering, Kyoto Institute of Technology, \\ Matsugasaki, Kyoto 606, Japan
}

(Received November 15, 1990)

\begin{abstract}
Structure and orientational behavior of two kinds of segmented poly(urethaneurea)s, SPUUs, having different soft segment molecular weights, were investigated by dynamic infrared absorption dichroism (DIR). DIR is a rheo-optical technique and a powerful tool to study orientational behavior of individual segments on multi-phase polymers under sinusoidal mechanical stimuli. SPUUs, being multi-block copolymers, consist of hard segment domains and soft segment matrix. Analysis of the temperature and frequency dependence of the dynamic strain-dichroic ratio coefficient, $L^{*}$ disclosed the following: (1) both the hard and soft segments are deformed synchronously (in-phase) with respect to applied strain, (2) the Arrhenius plot for the shift factor of $L^{*}$ is composed of two contributions, $\beta$ (the glass transition) and $\gamma$ dispersions (local motions related to methylene sequences). These observed dispersions were consistent with the corresponding mechanical dispersions, and (3) the hard segment of SPUU, having a larger soft segment molecular weight (TM-3), has a higher capability of orientation in response to external strain than that having a smaller soft segment molecular weight (TM-1).

KEY WORDS Dynamic Infrared Absorption Dichroism / Rheo-optics /

Segmented Poly(urethaneurea) / Phase Mixing /
\end{abstract}

It is well known that mechanical properties of polymeric materials are temperature and frequency dependent. The mechanical properties have often been investigated from the viewpoint of structure. Rheo-optics is one of the most powerful tools to reveal the relationship between microscopic structure and macroscopic properties. ${ }^{1}$ There are several techniques in rheo-optics, such as, dynamic $\mathrm{X}$-ray diffraction, dynamic light scattering, dynamic birefringence, and dynamic infrared dichroism. These techniques provide information on orientation of crystals, amorphous chains, and some particular polymer segments as well as mechanical response signals as a function of the applied strain.

Dynamic X-ray diffraction is one of the most appropriate techniques to get information about the deformation of the crystalline phase in semicrystalline polymers. ${ }^{2,3}$ The relationship between deformation mechanism and mechanical dispersion of polymers having a spherulite textures is discussed by this technique..$^{4-7}$ In addition, information about amorphous phases is also obtained indirectly by combination with a dynamic birefringence measurement. ${ }^{4,7}$ However, dynamic X-ray diffraction itself is limited to a study on crystal orientation in crystalline polymers. Dynamic birefringence gives only total optical anisotropy of a system and cannot be applied to multicomponent systems.

In the case of dynamic infrared absorption dichroism (DIR), one can focus several specific absorption bands of interest by choosing wave number. Therefore DIR can be used for many

\footnotetext{
* To whom correspondence should be addressed.
} 
kinds of polymers including multi-component systems irrespective of crystallinity. In addition, it provides some information on interactions between different atomic groups, such as hydrogen bonds, as a chemical shift in an infrared absorption spectrum. Nomura, et $a l .^{8,9}$ applied the DIR technique to low density polyethylene and discuss differences in dynamic orientation and mechanical behavior of crystalline and non-crystal region. They reported that the only crystalline phase had quadrature components in the $\alpha$ mechanical dispersion region.

Recently, Siesler ${ }^{10-16}$ discussed the relationship between the molecular orientation and stress-strain curve in natural rubber, polyurethane, and so on, by means of Fourier transform infrared spectroscopy coupled with a mechanical stretching device. He observed a drastic increment of stress on crystallization and attributed this to strain-induced crystallization.

Segmented poly(urethaneurea) (SPUU) is a multi-block copolymer consisting of hard and soft segments, and it is noted as industrial and medical material because of its excellent mechanical property and antithrombogenicity. The hard segments form domains and play as physical crosslink points. The soft segments, on the other hand, have an elastic nature at ambient temperature. Some of the characteristic properties of SPUU described above are due to the presence of a microphase separated structure.

A hydrogen bond is formed between a proton donor and acceptor. In the case of SPUU, the proton donor is the $\mathrm{N}-\mathrm{H}$ group of urea or urethane linkage and the proton acceptor is the oxygen of either urea carbonyl, urethane carbonyl or ether groups. The mechanical properties of SPUU are influenced considerably by the two phase structure and state of hydrogen bonds.

In previous papers, ${ }^{17,18}$ we discussed the structural change of SPUU during a fatigue process at both molecular and superstructure (spherulite) levels, and reported that phase mixing occurred with increasing fatigue time. It was also revealed that the hard segment domains were dominantly fatigued compared with the soft segment matrix by loading repetitive sinusoidal strains. ${ }^{19}$

In this paper, we discuss the dynamic orientation behavior of the hard and soft segments and deformation mechanism of SPUU over a wide temperature and time range.

\section{DEFINITION OF DYNAMIC STRAIN-OPTICAL COEFFICIENT}

Although there are many kinds of dynamic strain-optical coefficients, ${ }^{4}$ here we briefly describe the dynamic strain-optical coefficient obtained from dynamic infrared absorption dichroism.

When a polymer film was subjected to sinusoidal strain, the resulting stress $\sigma(t)$ may vary sinusoidally with the phase difference angle $\delta$ with respect to the dynamic bulk strain $\varepsilon(t)$. These can be generally described as follows:

$$
\begin{gathered}
\varepsilon(t)=\varepsilon_{0}+\Delta \varepsilon \exp (i \omega t) \\
\sigma(t)=\sigma_{0}+\Delta \sigma \exp [i(\omega t+\delta)]
\end{gathered}
$$

where $\varepsilon_{0}$ and $\delta_{0}$ are the static components of the strain and stress, respectively, and $\Delta \varepsilon$ and $\Delta \sigma$ are, respectively, the dynamic amplitudes of the strain and stress. $\omega$ represents angular frequency.

Defining parallel and perpendicular absorbances with respect to the strain direction as $A_{\|}$and $A_{\perp}$, respectively, the dichroic ratio is expressed by

$$
D=A_{\perp} / A_{\|}
$$

In the context of linear viscoelasticity, one expects that the dichroic ratio may be simply expressed by eq 4, which is similar to the mechanical response, stress, as shown in eq 2 ,

$$
D(t)=D_{0}+\Delta D \exp [i(\omega t+\alpha)]
$$


where $D_{0}$ and $\Delta D$ are the static and dynamic components of the dichroic ratio, respectively, and $\alpha$ is the phase angle between the strain and ratio.

The complex dynamic modulus $E^{*}$ is defined in terms of the strain and the stress as follows,

$$
\begin{aligned}
E^{*} & =\frac{\delta \sigma(t)}{\delta \varepsilon(t)} \\
& =E^{\prime}(\omega)+i E^{\prime \prime}(\omega)
\end{aligned}
$$

where $E^{\prime}(\omega)$ and $E^{\prime \prime}(\omega)$ denote the real and imaginary parts of the complex dynamicmodulus, and are called the storage and loss moduli, respectively. Likewise, a similar formulation may be used from the strain and dichroic ratio, as follows,

$$
\begin{aligned}
L^{*} & =\frac{\delta D(t)}{\delta \varepsilon(t)} \\
& =L^{\prime}(\omega)+i L^{\prime \prime}(\omega)
\end{aligned}
$$

The $L^{\prime}(\omega)$ and $L^{\prime \prime}(\omega)$ denote the real and imaginary components of the complex dynamic strain-dichroic coefficients, respectively. The physical meaning of $L^{\prime}$ is the capability of orientation since it is defined as the slope of the tangent in the variation of the dichroic ratio against stretching.

\section{EXPERIMENTAL}

\section{Samples}

SPUUs consisting of poly(tetramethylene glycol) (PTMG), 4,4'-diphenylmethane diisocianate (MDI), and 1,2-propylene diamine (PDA) were used. Table I shows the chemical composition and chemical structure of SPUUs. The molar ratio of MDI, PTMG, and PDA is $2: 1: 1$. Two kinds of specimens, which differed in the soft segment molecular weight, coded TM-1 and TM-3 as shown in Table I, were studied. Dimethyl formamide was used as a casting solvent. Two kinds of films having different thicknesses, about 10 and $300 \mu \mathrm{m}$, were prepared. The thin films were used for DIR measurements and thick films for
Table I. Chemical composition and chemical structure of segmented poly(urethaneurea)

Chemical composition of SPUU

\begin{tabular}{cccc} 
Sample & $\begin{array}{c}\text { Soft segment } \\
\text { PTMG }\left(M_{n}\right)\end{array}$ & MDI:PTMG:PDA & Solvent \\
\hline TM-1 & 856 & $2: 1: 1$ & DMF \\
TM-3 & 1343 & & \\
\hline
\end{tabular}

Chemical structures of SPUU

Soft segment (PTMG-MDI)

$$
\begin{aligned}
& {\left[\mathrm{O}+\mathrm{CH}_{2} \mathrm{CH}_{2} \mathrm{CH}_{2} \mathrm{CH}_{2} \mathrm{O}+\mathrm{C}-\mathrm{NH} \text { O } \mathrm{CH}_{2} \text { (O) }-\mathrm{NHC}_{m}\right.} \\
& m=5
\end{aligned}
$$

mechanical measurements. Details of the sample preparation are described in ref 17 .

\section{Dynamic Mechanical Measurements}

Dynamic mechanical properties were measured with a Rheospectoler Model DVE-V4 (Rheology Co., Ltd., Kyoto). By applying a synthesized strain composed of the basic wave of frequency of $0.15 \mathrm{~Hz}$ and its higher harmonics up to $19.2 \mathrm{~Hz}$, dynamic mechanical data were obtained by scanning temperature from -100 to $100^{\circ} \mathrm{C}$ at a heating rate of $4^{\circ} \mathrm{Cmin}^{-1}$.

\section{Static Infrared Absorption Measurements}

Static infrared absorption measurements were made on a FIRIS 100 Fourier transform infrared spectrometer, Fuji Electric Co., operated with a FASMIC G500/32 mini computer. In order to estimate segmental orientation, polarized infrared absorption spectra for unstretched and stretched films $(0$ to $250 \%$ elongation) were measured in the temperature range from 0 to $80^{\circ} \mathrm{C}$ under 
the condition of $4 \mathrm{~cm}^{-1}$ of resolution and at least 32 times the accumulation. Sample size was $40 \mathrm{~mm}$ long and $10 \mathrm{~mm}$ wide and gauge length was fixed at $30 \mathrm{~mm}$. A peak resolving procedure on the obtained spectra was used because several absorption bands overlapped each other.

\section{Dynamic Infrared Absorption Measurement}

A dynamic infrared absorption dichroism measuring system (DIR), developed by Nomura et $a l .{ }^{20}$ was used. A detailed description about the apparatus is reported elsewhere. ${ }^{9}$

Sample size was $40 \mathrm{~mm}$ long and $20 \mathrm{~mm}$ wide. Gauge length was $30 \mathrm{~mm}$. Dynamic infrared dichroism measurements were carried out under the $30 \%$ static strain and $7 \%$ dynamic strain in the temperature range from -10 to $80^{\circ} \mathrm{C}$ and in the frequency range from 1.22 to $0.00477 \mathrm{~Hz}$. Sinusoidal repetitive strain of $2.44 \mathrm{~Hz}$ in frequency was loaded onto a sample film for 30 minutes in advance to data acquisition so as to stabilize the specimens against mechanical agitation.

Figure 1 shows the infrared survey spectrum of TM-1 at $20^{\circ} \mathrm{C}$ from 2000 to $4000 \mathrm{~cm}^{-1}$. The absorption peak at $3315 \mathrm{~cm}^{-1}$ is assigned to the hydrogen-bonded $\mathrm{NH}$ stretching in the hard segment, $v(\mathrm{NH})$, and the absorption band located at $2940 \mathrm{~cm}^{-1}$ is due to the stretching of methylene groups in PTMG sequence, $v_{\mathrm{a}}\left(\mathrm{CH}_{2}\right)$. These peaks were used as probes of orientation analysis. We used peak intensities instead of areas of absorption bands because it was impossible to evaluate the areas of absorption bands due to restriction of the

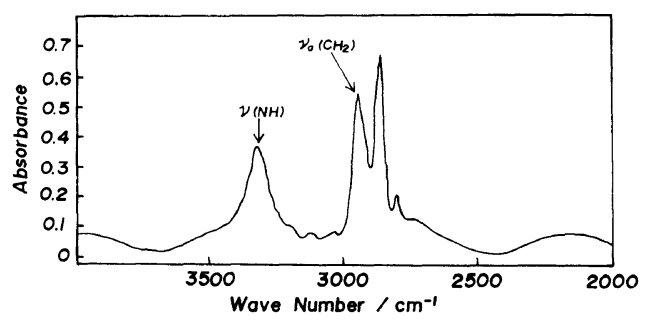

Figure 1. Infrared survey spectrum of TM-1 in the wave number region from 4000 to $2000 \mathrm{~cm}^{-1}$. apparatus employed here. However, the peak intensities studied were strong enough to permit assuming that it would not bring fatal error to the analysis of segmental orientation.

\section{RESULTS AND DISCUSSION}

\section{Thermal and Dynamic Mechanical Properties}

Figure 2 shows the differential scanning calorimeter (DSC) thermograms obtained with a DSC (DSC model 8230, Rigaku Denki Co.) for TM-1 and TM-3. The glass transition temperature of the soft segment, $T_{\mathrm{g}, \mathrm{s}}$, and melting temperature of the hard segments, $T_{\mathrm{m}, \mathrm{h}}$, were detected for both segments as shown in the figure. In addition, TM-3 having a larger soft segment molecular weight shows an endothermic peak due to melting of soft segments, $T_{\mathrm{m}, \mathrm{s}}$ around $-5^{\circ} \mathrm{C}$.

The crystallinity of the soft segment in TM-3 was estimated by the following method. A commercial PTMG, Terathane 1000, du Pont, was used as a reference sample, since the molecular weight of Terathane was $c a .1000$, which was close to that of the soft segment of TM-3. The value of the heat of fusion of Terathane was $22.3 \mathrm{kcal} \mathrm{mol}^{-1}$. By assuming $100 \%$ crystallinity for Terathane and soft segment content in TM-3 being $72 \mathrm{wt} \%$, the degree of crystallinity of the soft segment in TM-3 was estimated to be $c a$. $8 \%$. This value gives the lower limit for the crystallinity of the soft segments because it is apparent that

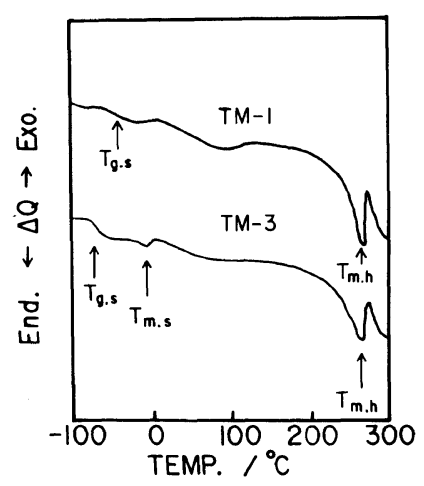

Figure 2. DSC curves of the TM series. 
the crystallinity of the reference sample is less than $100 \%$. It is surprising to note that the crystallinity is remarkably low. Only a small portion of PTMG (about 8\%) was crystallized in TM-3. This indicates that formation of the block sequence with MDI lowers significantly the capability of the crystallization of PTMG.

This shows that the soft segment hardly crystallize even below $T_{\mathrm{m}, \mathrm{s}}$, and soft segment crystals do not play important roles as physical crosslink points.

Temperature dependences of the storage and

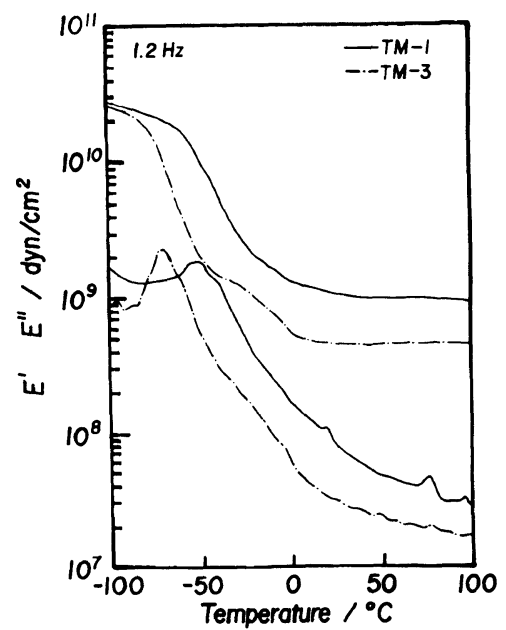

Figure 3. Temperature dependence of storage modulus, $E^{\prime}$, and loss modulus, $E^{\prime \prime}$, for TM-1 (solid line) and TM-3 (dotted line).

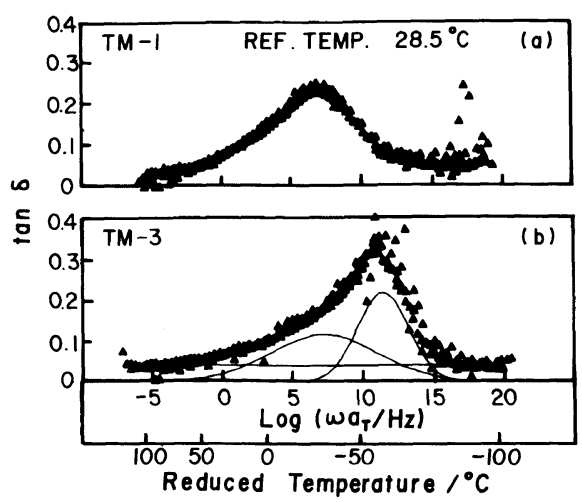

Figure 4. Master curves of $\tan \delta_{\mathrm{TO}}\left(\omega a_{\mathrm{T}}\right)$ for specimens (a) TM-1 and (b) TM-3 reduced to a reference temperature of $30^{\circ} \mathrm{C}$. loss moduli, $E^{\prime}$ and $E^{\prime \prime}$, for TM-1 and TM-3 are shown in Figure 3. TM-1 and TM-3 have the glass transition region around $-50^{\circ} \mathrm{C}$, and $-70^{\circ} \mathrm{C}$, respectively, which are in accordance with the DSC results.

Figures 4(a) and (b) show master curves for mechanical loss tangent, $\tan \delta$, of TM series, all the data fragments obtained at different temperatures were reduced with respect to a common reference temperature of $28.5^{\circ} \mathrm{C}$ by horizontally shifting the individual curves. A similar mechanical master curve for $\tan \delta$ was observed by DIR measurement, which was consistent with Figures 4(a) and (b) in both magnitude and $\omega a_{\mathrm{T}}$ dependence.

Since the master curve for TM-3 is not symmetric, it is expected that more than one mechanical relaxation mechanism is present. The Arrhenius plot for TM-3 consisting of two lines means that TM-3 has two mechanical relaxation mechanisms. Hence, by assuming a Gaussian function for the individual relaxation functions, we resolved this master curve into two components. The solid curves in Figure 4(b) represent the individual components. The straight line is the base line. Two peaks appeared around $\log \omega a_{\mathrm{T}}=7$ and 11. The frequency at maximum in the dispersion curve located at the high frequency side corresponds

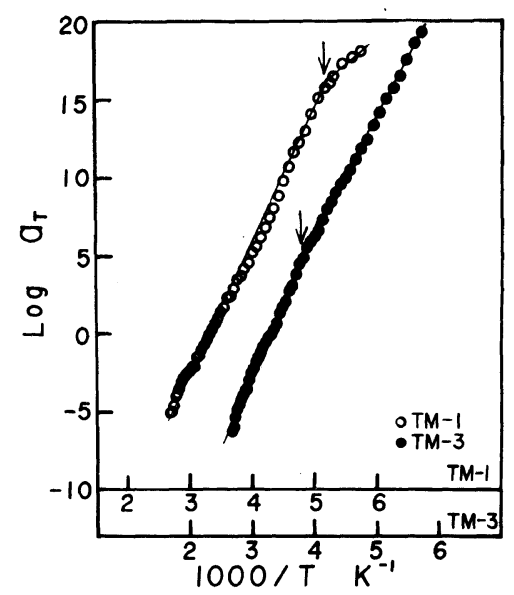

Figure 5. Temperature dependence of horizontal shift factors for making the mechanical master curves of specimens TM-1 and TM-3. 
to $-60^{\circ} \mathrm{C}$ which is close to the glass transition temperature of TM-3. Therefore, this component is assigned to the $\beta$ dispersion. The frequency of dispersion on the low frequency side corresponds to $-30^{\circ} \mathrm{C}$. Kimura and Ishihara et $\mathrm{al}^{21}$ reported the appearance of crystalline dispersion of the soft segment, $\alpha_{s}$, when PTMG molecular weight is large. In this case, since the endothermic peak due to the melting of PTMG crystals was observed, this dispersion may be assigned to the crystalline dispersion of the soft segment.

Figure 5 shows the reciprocal temperaturedependence of the horizontal shift factors, $a_{T}$ obtained from superposition of $\tan \delta$. It was found that each of the Arrhenius plots for both specimens consists of two straight lines, the crossover points being indicated by arrows. This may mean that SPUUs have two mechanical relaxation mechanisms in the range of the temperature and frequency studied here. The mechanical relaxation mechanism at the high frequency side of TM-1 may be assigned to the $\gamma$ dispersion related to the local motion of polymer chains. On the other hand, the reduced temperature at maximum in $\tan \delta$ is close to the glass transition temperature obtained from DSC, and its activation energy is also consistent with the literature value of the $\beta$ dispersion of polyurethane. Because of the above reasons, we assigned the mechanical dispersions as $\beta$ and $\gamma$ dispersions. The activation energies calculated from these slopes are listed in Table II.

\section{Static Infrared Absorption Dichroism}

Figures 6(a) and (b) show the elongation

Table II. Apparent activation energies of various mechanical dispersions

\begin{tabular}{cccc}
\hline & \multicolumn{4}{c}{ Activation energy $/\left(\mathrm{kcal} \mathrm{mol}^{-1}\right)$} \\
\cline { 2 - 4 } Specimens & $\alpha_{\mathrm{s}}$ & $\beta$ & $\gamma$ \\
\hline TM-1 & - & 36.8 & 18.9 \\
TM-3 & 41.9 & 33.9 & - \\
\hline
\end{tabular}

dependence of the dichroic ratio of $\mathrm{NH}$ band. Figures 7(a) and (b) show that of the methylene groups. Because the transition moments of both bands are roughly perpendicular to the corresponding backbone chains and the dichroic ratio is defined by eq $3, D>1$ and $D<1$ mean positive and negative orientations of the segments, respectively. As shown in

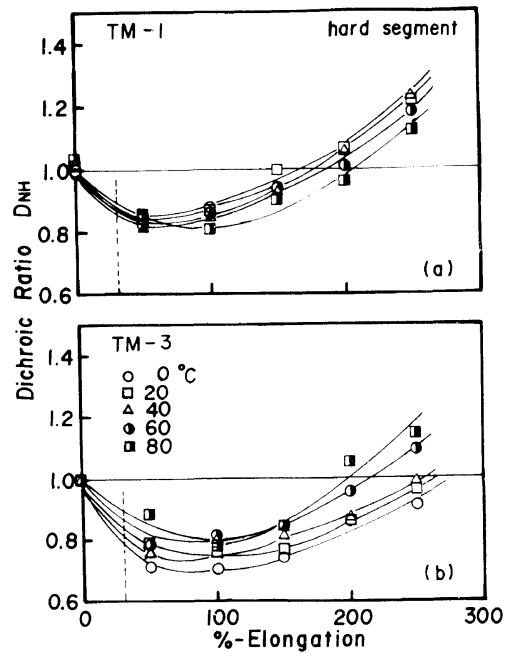

Figure 6. Elongation dependence of dichroic ratio in the hard segment: (a) for the TM-1 series; (b) for the TM-3 series.
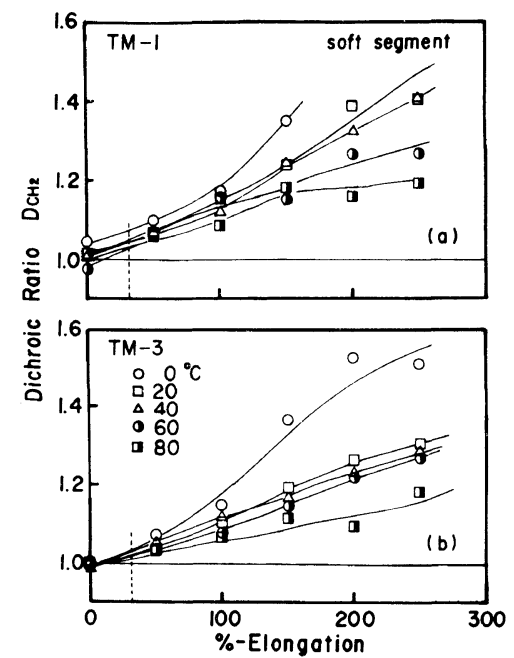

Figure 7. Elongation dependence of dichroic ratio in the soft segment: (a) for the TM-1 series; (b) for the TM-3 series. 
Figure 6, the hard segment orients negatively at low elongation. This can be explained in terms of the lamellar model ${ }^{22,23}$ or spherulite deformation model. ${ }^{17,18,24}$ The hard segment for TM-1 shows the largest negative orientation at around $50 \%$ elongation, and the magnitude of the negative orientation hardly depends upon temperatures. On the other hand, TM-3 has the largest negative orientation at about $100 \%$ elongation, and the tendency of negative orientation decreases with increasing temperature. This difference in the hard segment orientation behavior between TM-1 and TM-3 indicates differences in aggregation states between the two.

$\%$-Elongation dependence of the dichroic ratio for the soft segments is rather simple compared to the hard segments as shown in Figures 7(a) and (b). The soft segment orients monotonously with increasing \%-elongation. The capability of orientation of the soft segment, however, seems to decrease by elevating the temperature. This will be discussed later.

The broken lines in Figures 6 and 7 indicate $30 \%$ elongation where the static strain is applied in the DIR measurements. The dichroic ratio has a tendency to be less dependent on
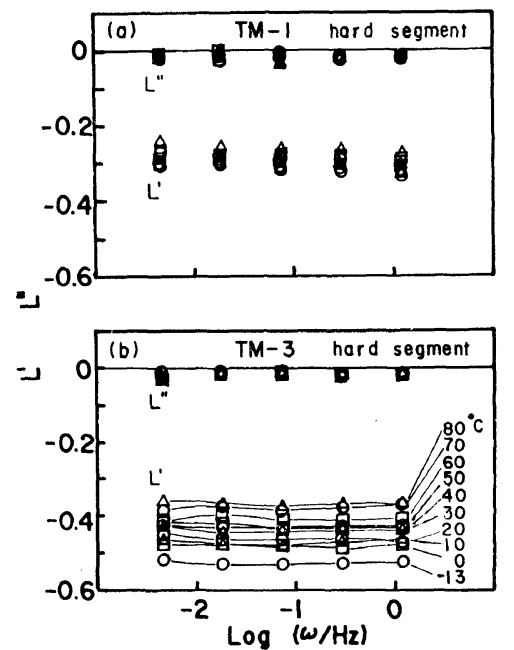

Figure 8. Frequency dependence of $L^{\prime}$ and $L^{\prime \prime}$ for the hard segment at various temperatures: (a) TM-1; (b) TM-3. elongation with increasing temperature in this low elongation region. This means that the external strain becomes less transmitted at the molecular level at higher temperature.

\section{Dynamic Infrared Absorption Dichroism}

Figures 8(a) and (b) show the frequency dependence of the real and imaginary components of the complex dynamic strain-dichroic ratio coefficient, $L^{\prime}$ and $L^{\prime \prime}$, for the hard segment of TM-1 and TM-3 at various temperatures. No optical dispersion seems to be present in this temperatures and frequency range. Because $L^{\prime \prime}$ is much smaller than $L^{\prime}$ and is almost close to zero for both TM-1 and TM-3, the hard segments show in-phase orientation behavior against the applied strain. $L^{\prime}$ of TM-1 does not depend either on temperature or frequency. On the other hand, that of TM-3 does depend temperature; i.e., it decreases with temperature, while its frequency dependence is negligible. In addition, the magnitude of $L^{\prime}$ of TM-3 is larger than that of TM-1, indicating that the hard segments of TM-3 orient easily compared with that of TM-1. This corresponds to the static infrared dichroism measurement as shown in Figure 6(a).

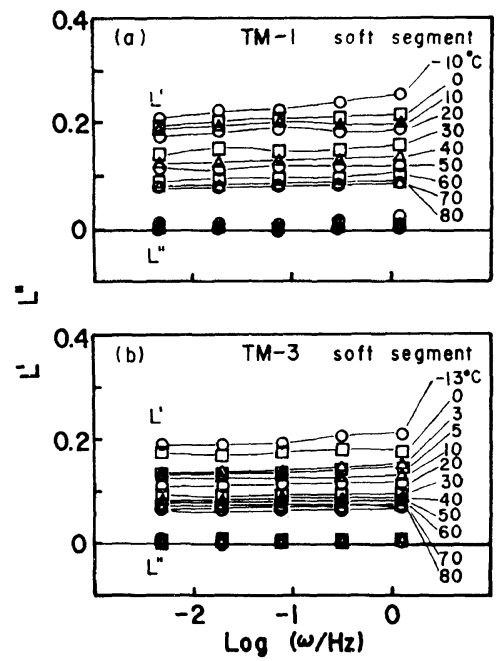

Figure 9. Frequency dependence of $L^{\prime}$ and $L^{\prime \prime}$ for the soft segment at various temperatures: (a) TM-1; (b) TM-3. 
Figures 9(a) and (b) show the frequency dependence of $L^{\prime}$ and $L^{\prime \prime}$ for the soft segments of TM-1 and TM-3 at various temperatures. It was found that the soft segments for both specimens also show in-phase deformation behavior against the external strain since $L^{\prime \prime}$ of the soft segment is almost zero and flat. Although the appearance of optical dispersion due to crystalline melting is expected, it is not observed in these figures. Because the degree of crystallinity being $8 \%$ is very low, the behavior of amorphous chains may be an important contribution to dynamic infrared dichroism. $L^{\prime}$ of the soft segments both TM-1 and TM-3 decreases with increasing temperature or decreasing frequency. This indicates that the orientational ability of the soft segments decreases at high temperature and low frequency. Furthermore, since the magnitudes of $L^{\prime}$ for both specimens are nearly equal, the soft segments have similar orientational ability. This is also consistent with the static infrared measurement shown in Figure 6(b).

Since $L^{\prime}$ of the soft segments shows temperature and frequency dependence, we carried out temperature-time superposition, and made master curves as shown in Figures 10(a) and (b). The reduced temperature was chosen to be $30^{\circ} \mathrm{C}$. The superposition was conducted by shifting each curve in Figures 9(a) and (b) with a horizontal shift factor $\log O_{\mathrm{T}}$. As can be seen in the figures, the master curves of TM-1 and TM-3 increase on the high frequency side. These results suggest that there may be some dispersion on the high frequency side.

Figure 11 shows the horizontal shift factors obtained from the temperature-time superposition of $L^{\prime}$ for TM-1 and TM-3 plotted as a function of reciprocal absolute temperature. The Arrhenius plots of the horizontal shift factors of both TM-1 and TM-3 give straight lines. The apparent activation energies calculated from these slopes are 38.3 and $42.7 \mathrm{kcal}$ $\mathrm{mol}^{-1}$ for TM-1 and TM-3, respectively, and are roughly close to the values of mechanical

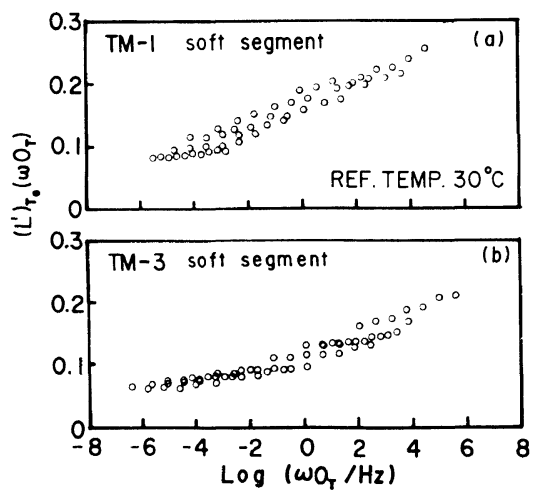

Figure 10. Master curves of $L^{\prime}$ for the soft segment reduced to a reference temperature of $30^{\circ} \mathrm{C}$ : (a) $\mathrm{TM}-1$; (b) TM-3.

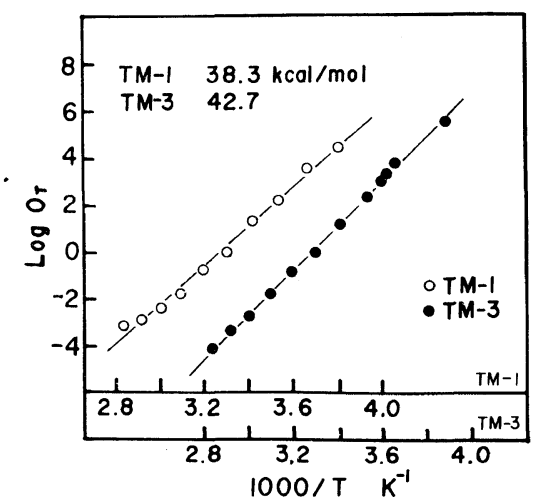

Figure 11. Temperature dependence of horizontal shift factors for making the optical master curves of specimens: O, TM-1; 0 , TM-3.

dispersion appeared at the higher temperature side.

\section{Deformation Mechanism}

We discuss here the deformation mechanism of SPUU based on the results of the static and dynamic IR measurements. In general, in the case of elastomers stretched uniaxially at a given ratio, the degree of orientation does not change with temperature except for a slight change due to thermal expansion of the elastomer although stress changes linearly with absolute temperature. In the case of SPUU, however, the dichroic ratio has a tendency to decrease to unity by elevating the tempera- 
ture. This behavior is explained by nonpermanent crosslinks in SPUU, such as hard segment domains, hydrogen bonds, and entanglements. As shown in Figure 9, the soft segments orient positively according to the external strain just as conventional elastomers. $L^{\prime}$, a measure of the capability of orientation, decreases with temperature. This strongly indicates that the number of physical crosslinks decreases and the effective length of the soft segment chains increases, resulting in lowering of efficiency of transmission of the external strain down to the molecular level. Dissociation of hydrogen bonds in both the hard segment domains and soft segment matrix seems to be mainly responsible for the decrease in the number of crosslinks.

In the case of hard segment orientation, we need to recall the superstructures of SPUU proposed by several workers, since hard segment orientation cannot be understood without knowledge of superstructure. Negative orientation behavior of the hard segments in segmented poly(urethaneurea)s was often observed and several structure models proposed in order to account for this negative orientation. It has already been published that both specimens used in this experiment have spherulitic structures ${ }^{17,18}$ and that TM-3 has well developed hard segment domains. ${ }^{28} \mathrm{~W}$ hen the spherulite is deformed, the well developed hard segment domains are not destroyed but deformed just as we deform a rubber ball, and they exhibit a large negative orientation. On the other hand, in the case of TM-1, the hard segment domain is destroyed by stretching because the PTMG sequence is short and the cohesive force in the hard segment domains is weak. As shown in Figures 6(a), (b), the largest negative orientation for TM-3 is larger than that for TM-1 (see Figure 6(a), (b)) because of the above described reason. It is noteworthy that we must also consider the annealing effect. Kotani ${ }^{29}$ indicated that the trend of negative orientation was increased by annealing. Since negative orientation is lowered by increasing temperature, as shown in Figure 4, we can neglect the annealing effect.

It has been already reported that the cohesive force of the hard segment domain of TM-3 drastically decreases with increasing temperature and that of TM-1 hardly changes. ${ }^{28}$ The hard segment domains of TM-1 having the lower cohesive force is easily destroyed by deformation. This leads to an increased dichroic ratio and $L^{\prime}$. However, since the cohesive force of TM-1 does not show temperature dependence, ${ }^{28}$ no change of dichroic ratio or $L^{\prime}$ was observed.

\section{CONCLUSIONS}

Static and dynamic infrared absorption dichroism measurements were performed in order to study the deformation mechanism of segmented poly(urethaneurea)s over a wide range of temperatures and frequency and the following facts were disclosed:

1) The orientational behavior of hard and soft segments for TM-1 and TM-3 is in-phase against external strain.

2) The capability of orientation for the soft segment decreases at high temperature or low frequency. This is due to increase in the effective length of the soft segments by lowering the number of effective crosslinks in the soft segment matrix at high temperature or low frequency.

3) The capability of the negative orientation of the hard segments in TM-3 decreases with increasing temperature but that of TM-1 does not depend either temperature or frequency. This can be explained based on differences in cohesive force in hard segment domains.

Acknowledgments. The financial support from Resources and Environment Protection Research Laboratory, NEC. Corp., Kawasaki, Japan, is gratefully acknowledged.

This work was supported by a Grant-in-Aid for Scientific Research (No. 01-460-229) from 
the Ministry of Education, Science, and Culture of Japan.

\section{REFERENCES}

1) R. S. Stein, Polym. J., 17, 289 (1985).

2) K. Saijo, S. Suehiro, and T. Hashimoto, Polym. Prepr. Jpn., 38, 1117 (1989).

3) S. Suehiro, K. Saijo, Y. Ohta, T. Hashimoto, and H. Kawai, Anal. Chim. Acta, 189, 41 (1986).

4) A. Tanaka, E. P. Chang, B. Delf., I. Kimura, and R. S. Stein, J. Polym. Sci., Polym. Phys. Ed., 11, 1891 (1973).

5) T. Kyu, S. Suehiro, S. Nomura, and H. Kawai, $J$. Polym. Sci., Polym. Phys. Ed., 18, 951 (1980).

6) T. Kyu, M. Yamada, S. Suehiro, and H. Kawai, Polym. J., 12, 809 (1980).

7) K. Fujita, H. Niwa, S. Nomura, and H. Kawai, $J$. Polym. Sci., Polym. Phys. Ed., 21, 1713 (1983).

8) S. Nomura, Sen-i to Kougyou, 41, 450 (1985).

9) S. Nomura, M. Nakamichi, Y. Yamamoto, M. Ohgushi, T. Yamamoto, T. Soen, and M. Shibayama, in preparation.

10) H. W. Siesler, Polym. Bull., 9, 382 (1983).

11) H. W. Siesler, Polym. Bull., 9, 471 (1983).

12) H. W. Siesier, Polym. Bull., 9, 557 (1983).

13) H. W. Siesler, Adv. Polym. Sci., 65, 1 (1984).

14) H. W. Siesler, Colloid Polym. Sci., 262, 223 (1984).

15) H. W. Siesler, Pure Appl. Chem., 57, 1603, (1985).

16) H. W. Siesler, Ber Bunsenges Phys. Chem., 92, 641
(1988).

17) M. Shibayama, T. Kawauchi, T. Kotani, S. Nomura, and T. Matsuda, Polym. J., 18, 719 (1986).

18) M. Shibayama, Y. Ohki, T. Kotani, and S. Nomura, Polym. J., 19, 1067 (1987).

19) T. Yamamoto, M. Shibayama, and S. Nomura, Rep. Prog. Polym. Phys. Jpn., 30, 541 (1987).

20) S. Nomura, M. Nakamichi, M. Morikawa, M. Kurokawa, and T. Soen, Rep. Prog. Polym. Phys. Jpn., 26, 495, (1983).

21) H. Ono and I. Kimura, "Kagakuzoukan," No. 58, Kagakudoujin, Kyoto, 1973, p 185.

22) F. Hoffman and R. Bonart, Makromol. Chem., 184, 1529 (1983).

23) R. Bonart., J. Macromol. Sci. Phys., 132, 115 (1968).

24) I. Kimura, H. Ishihara, H. Ono, N. Yoshihara, S. Nomura, and H. Kawai, Macromolecules, 7, 355, (1974).

25) S. B. Lin, K. S. Hwang, S. Y. Tsay, and S. L. Cooper, Colloid Polym. Sci., 263, 128 (1985).

26) H. Ishihara, I. Kimura, K. Saito, and H. Ono, $J$. Macromol. Sci.-Phys., B10, 591 (1974).

28) C. B. Wang and S. L. Cooper, Macromolecules, 16, 775 (1983).

27) T. Yamamoto, M. Shibayama, and S. Nomura, Polym. J., 21, 895 (1989).

29) T. Kotani, M. S. thesis, submitted to Department of Textile Engineering, Kyoto Institute of Technology Kyoto (1987). 